\title{
Estimation of energy expenditure using accelerometers and activity-based energy models-validation of a new device
}

\author{
Sascha Härtel • Jens-Peter Gnam • Simone Löffler • \\ Klaus Bös
}

Received: 9 July 2010 /Accepted: 22 November 2010 / Published online: 2 December 2010

(C) European Group for Research into Elderly and Physical Activity (EGREPA) 2010

\begin{abstract}
Over the last few years, the estimation of energy expenditure with accelerometers has become more and more accurate due to improvements in sensor technology. Significant enhancement could be reached by model-based estimation regarding different activity types. The kmsMove-sensor (movisens GmbH, Karlsruhe, Germany) is a device that is used to compute human energy expenditure using motiondependent calculation models. It is outfitted with an accelerometer to measure body acceleration during certain movements and activities. To validate its accuracy, the sensor was compared to indirect calorimetry as criterion measure. For this experiment, nine subjects (all males, age $46.4 \pm 10.9$ years, 28 64 years) were equipped with the kmsMove-sensor as well as a portable indirect calorimeter and their energy consumption was measured over a time period of $100 \mathrm{~min}$. Additionally, the energy consumption of seven out of the above-mentioned nine subjects was measured over an average of $7 \mathrm{~h}$. The measurements took place in a rehabilitation clinic, where the subjects completed their regular daily rehabilitation activities. An analysis of the data revealed ICCs between the kmsMovesensor and indirect calorimetry for the time period of $100 \mathrm{~min}$ of $0.82(0.38-0.96 ; p=0.003)$ and for an averaged measuring time of $7 \mathrm{~h}$ of $0.81(0.22-0.97 ; p=0.01)$. Furthermore, a Bland-Altman analysis for the time period of $100 \mathrm{~min}$ led to
\end{abstract}

\footnotetext{
S. Härtel · J.-P. Gnam · S. Löffler

House of Competence-RG hiper.campus,

Karlsruhe Institute of Technology (KIT),

Karlsruhe, Germany

S. Härtel $(\bowtie) \cdot$ J.-P. Gnam · K. Bös

Institute of Sports and Sports Science,

Karlsruhe Institute of Technology (KIT),

Engler-Bunte-Ring 15,

76131, Karlsruhe, Germany

e-mail: Sascha.Haertel@kit.edu
}

a difference of the means of $4.3 \mathrm{kcal}$ (limits of agreement: -94.3 and $102.9 \mathrm{kcal}$ ) and for the time period of an average of $7 \mathrm{~h}$ to $-14.0 \mathrm{kcal}$ (limits of agreement: -320.0 and $292.0 \mathrm{kcal})$. These findings indicate that the kmsMovesensor is an appropriate measuring device with relatively good accuracy to assess human energy expenditure in rehabilitation patients. However, this study has some limiting aspects (small sample size, artificial setting) which could influence validity.

Keywords Accelerometer - Energy expenditure - Activity classification · Validity $\cdot$ Indirect calorimetry

\section{Introduction}

Assessment of the physiological parameter "energy expenditure" has become more and more important, not only under research aspects but also in matters of illness prevention and health promotion. Hence, Paffenbarger et al. (1993) recommend an optimum of 300-400 kcal of daily energy expenditure from physical activity to achieve healthprotecting effects [1]. Thus, there is a certain need to assess energy expenditure of free-living individuals in everyday life and to develop appropriate energy expenditure measurement devices, so that people can be enabled to easily assess their energy expenditure and thus can control for adequate physical activity. There are a handful of existing measurement techniques to assess energy expenditure but only a few of them are appropriate to apply to measurements in everyday life [2]. Indirect calorimetry via expiratory gas exchange measurement is often referred to as a "gold standard" for measuring energy expenditure. In natural settings or field studies, indirect calorimetry is not always an appropriate measurement technique because 
subjects have to wear a respiratory mask and are therefore limited in performing their activities of daily living. Accelerometers are easy to handle, the subjects are not interfered by the measurement, and registration of physical activity is nearly free of artifacts [3]. A disadvantage to current accelerometers is that they only use one algorithm to compute the energy expenditure from the captured raw data. No single regression equation is able to accurately predict energy expenditure for all kinds of activities [4]. This is mainly due to the variety and complexity of diverse activities and movements and their relation to energy expenditure. The weaknesses in assessing accurate energy expenditure by accelerometry are mainly due to the applied algorithms, and to a much lesser extent, due to the technology of the accelerometers [5]. Therefore, it is necessary to improve the calculation of energy expenditure by using different regression equations for different kinds of activities. The kmsMove-sensor is a new device which captures the movements of its user on the basis of accelerations. The sensor is capable of allocating the different movements to certain categories of activity. According to the category of activity, the sensor chooses one of five algorithms to calculate the energy expenditure based on the captured raw data (acceleration values). The aim of this study is to investigate how accurately energy expenditure can be assessed by the kmsMove-sensor. To assess the energy expenditure measurement accuracy of the kmsMove-sensor, the sensor was compared to indirect calorimetry as criterion measure.

\section{Methods}

\section{Procedure}

The study took place in the rehabilitation clinic Überruh in Isny, Germany. Fifteen patients wore both, the kmsMovesensor on the hip and the portable indirect calorimeter MetaMax 3B (Cortex Biophysik, Leipzig, Germany) and completed their regular daily rehabilitation activities. The general physical activities were bicycle ergometer training, gymnastics, walking, and hiking. Besides these activities, the patients' rehabilitation programs consisted of other kinds of activities like relaxation or psychological counseling. The measurements of all subjects started at 7:00 am and ended at 5:00 pm. Within that time span, there were breaks for breakfast and lunch and for changing clothes. The kmsMove-sensor recorded data without breaks while indirect calorimetry was paused for breaks. Afterwards, the test readings of the kmsMove-sensor and indirect calorimetry were synchronized. Due to a breakdown of one of the kmsMove-sensors and the disruption of the indirect calorimeters caused by cold weather conditions during outdoor activities, a loss of data occurred. These technical problems could not be solved in situ. Thus, the data for only nine patients could be analyzed, at least for a time period of $100 \mathrm{~min}$. From these nine patients, the recordings of seven patients last for an average of $7 \mathrm{~h}$.

\section{Participants}

The participants were patients of the rehabilitation clinic Überruh in Isny, Germany. They had been informed about the study in an oral presentation and provided informed consent on participating voluntarily. The remaining nine subjects of the original sample were all males at a mean age of $46.4 \pm 10.9$ years. They had a mean weight of $84.5 \pm$ $9.2 \mathrm{~kg}$ and a mean height of $177.7 \pm 8.1 \mathrm{~cm}$. The subjects were all treated for back pain, received no medication, and were free of any cardiovascular complications.

Measurement instruments

\section{kmsMove}

The kmsMove-sensor consists of a $5.3 \times 3 \times 2$-cm-sized body and can be fixed with a clip to the hip. Alternative fixation with a chest belt and wristband are also possible. During the measurements, the sensor was placed on the right side of the subjects' hip. The sensor is a three-axial acceleration sensor with a range of $\pm 8 \mathrm{~g}$, a resolution of $12 \mathrm{bit}$ and a sampling rate of $128 \mathrm{~Hz}$. The recorded data from the sensor, including raw data from the acceleration sensor, can be displayed on a computer when connected to it via USB cable. While being connected, the sensor can also be charged and additionally configured with a special software that is optimized to manage scientific studies with large numbers of participants. After downloading data from the sensor, the energy expenditure is calculated and stored in a CSV file. Energy expenditure is displayed in steps of $1 \mathrm{~s}$. Short time intervals allow monitoring spontaneous activities. The sensor offers an overall measuring time of 7 days. The calculation of energy expenditure is done in three steps: activity recognition, model selection, and calculation of energy expenditure. The recognition of different activities is based on the extraction of mathematical and statistical features of the raw acceleration signal. The features are calculated for each segment of $4 \mathrm{~s}$. Calculated features are, amongst others, maximum frequency, step count, and the number of mean crossings. These features are the input information of a decision tree which classifies the activity of the person. Activities that can be detected are rest (combination of lying, sitting, and standing); bicycle or ergometer; going upstairs; walking (combination of jogging, going downstairs, walking slow, normal, and fast); and unknown activity. According to 
information from the manufacturer, the decision tree was generated using data of approximately 100 subjects who performed daily life activities. The accuracy of the activity recognition algorithm is discussed by Jatobá et al. [6]. According to the detected activity, one of five different models is selected. The formula for the models which are used to calculate energy expenditure is:

$$
\begin{aligned}
\mathrm{EE}= & b_{0}+b_{1} \mathrm{EEAC}+b_{2} \mathrm{Age} \cdot \mathrm{EEAC}+b_{3} \text { Height } \cdot \text { EEAC } \\
& +b_{4} \text { Weight } \cdot \text { EEAC }
\end{aligned}
$$

For each activity, the model is built by a set of five coefficients $\left(b_{0}-b_{4}\right)$. The coefficients were generated using data of indirect calorimetry of the above-mentioned 100 subjects. EEAC is derived from the zero-mean acceleration signal $\left(a_{x}, a_{y}, a_{z}\right)$ in a segment of $1 \mathrm{~s}$ which consists of $N$ samples like:

EEAC $=\operatorname{smooth}\left(\frac{1}{N} \sum_{i=1}^{N} \sqrt{a_{x i}{ }^{2}+a_{y i}{ }^{2}+a_{z i}{ }^{2}}\right)$

EEAC is smoothed using a moving average filter. Different models are used because there are activities with high overall acceleration (EEAC) but low energy expenditure (EE) and on the other hand, there are activities with low overall acceleration but high energy expenditure. The use of different models compensates such over- or underestimations. By the use of models that are dependent on subject-specific parameters like age, height, and weight, no subject-specific calibration of the sensor was necessary $[6,7]$.

\section{Indirect calorimetry}

Indirect calorimetry is a procedure, which is based on a certain relationship between the combustion of substrates, the consumption of oxygen, the expiration of carbon dioxide, and the production of energy. For the combustion of substrates, a certain amount of oxygen is required and a certain amount of carbon dioxide is accumulated. From the relationship of oxygen uptake and carbon dioxide expiration, the metabolic respiratory quotient can be calculated. For every metabolic respiratory quotient there is a certain caloric equivalent, which expresses the relationship between the combustion of a certain substrate, the required oxygen volume, and the amount of energy which is produced. If the caloric equivalent and the consumed oxygen volume are multiplied, one obtains the energy expenditure for that certain time period [8].

The portable indirect calorimeter MetaMax 3B uses the breath-by-breath technique and is able to run up to $20 \mathrm{~h}$ without being connected to a PC via wireless telemetry technology. This system is lightweight, comfortable, and offers good mobility for the user. For calculating energy expenditure, the MetaMax 3B uses an RQ-based equation with an assumed protein utilization part of total energy production (15\%) according to Acheson [9].

\section{Statistical methods}

In order to investigate the agreement between the test readings of the kmsMove-sensor and indirect calorimetry, a two-way mixed, single measure, intraclass correlation (ICC $(3,1))$ was conducted. Therefore, the software SPSS Statistics 17.0 (SPSS Inc., Chicago, IL, USA) was applied. Furthermore, a Bland-Altman analysis was also performed to examine the agreement between the two devices. This has been done with Analyse-it for Microsoft Excel (Analyse-it Software, Ltd., Leeds, UK). The mean difference and the limits of agreement were calculated according to Bland and Altman [10]. The Bland-Altman analysis is a statistical procedure to check the agreement of two different measuring devices. Therefore, the measurement differences of both devices were plotted against their mean. Normally, these differences follow a
Table 1 Results of energy expenditure measurements over $100 \mathrm{~min}$

\begin{tabular}{lcccc}
\hline Subject number & Indirect calorimetry (kcal) & kmsMove-sensor (kcal) & $\begin{array}{l}\text { Difference } \\
\text { (kcal) }\end{array}$ & $\begin{array}{l}\text { Percentage } \\
\text { difference (\%) }\end{array}$ \\
\hline 1 & 373 & 361 & -12 & -3 \\
2 & 401 & 436 & +35 & +9 \\
3 & 578 & 578 & 0 & 0 \\
4 & 378 & 405 & +27 & +7 \\
5 & 509 & 468 & -41 & -8 \\
6 & 411 & 509 & +98 & +24 \\
7 & 490 & 494 & +4 & +1 \\
8 & 357 & 368 & +11 & +3 \\
9 & 579 & 496 & -83 & -14 \\
$\varnothing$ total (absolute & $453 \pm 88$ & $457 \pm 71$ & $35 \pm 35$ & $8 \pm 8$ \\
value) & & & \\
\hline
\end{tabular}


Table 2 Results of energy expenditure measurements over an average of $7 \mathrm{~h}$

\begin{tabular}{lcclcc}
\hline Subject number & $\begin{array}{l}\text { Measurement } \\
\text { duration (hours) }\end{array}$ & $\begin{array}{l}\text { Indirect } \\
\text { calorimetry (kcal) }\end{array}$ & $\begin{array}{l}\text { kmsMove- } \\
\text { sensor (kcal) }\end{array}$ & $\begin{array}{l}\text { Difference } \\
\text { (kcal) }\end{array}$ & $\begin{array}{l}\text { Percentage } \\
\text { difference (\%) }\end{array}$ \\
\hline 1 & 7.6 & 1,917 & 1,788 & -129 & -7 \\
2 & 7.2 & 1,595 & 1,614 & +19 & +1 \\
3 & 7.4 & 2,116 & 2,062 & -54 & -3 \\
4 & 7.5 & 1,503 & 1,610 & +107 & +7 \\
6 & 7.2 & 1,589 & 1,837 & +248 & +16 \\
8 & 7.5 & 1,856 & 1,631 & -225 & -12 \\
9 & 5.2 & 1,405 & 1,341 & -64 & -5 \\
$\varnothing$ total (absolute value) & $7.1 \pm 0.8$ & $1,711 \pm 256$ & $1,698 \pm 226$ & $121 \pm 156$ & $7 \pm 5$ \\
\hline
\end{tabular}

normal distribution and so $95 \%$ of the differences usually lie between the limits of \pm 2 standard deviations around the mean difference (bias) of the two devices. Those limits are the limits of agreement. If the differences of the two devices lie between the limits of agreement, the two devices can be used interchangeably [10].

\section{Results}

In Table 1 total energy expenditure values of each subject over a measuring time of $100 \mathrm{~min}$ are presented. As one can see from Table 1, the difference of the means of both measurement devices is only $4 \mathrm{kcal}$, with a very small overestimation of energy expenditure by the kmsMovesensor. The total averaged difference is $35 \mathrm{kcal}$ or $8 \%$.

Table 2 shows the test readings of both devices over an average of $7 \mathrm{~h}$. Unfortunately, there occurred a loss of data with subjects 5 and 7 , so their test readings were not long enough to be considered for a long time period. The test readings of the remaining seven subjects show a difference of the means of both devices of $13 \mathrm{kcal}$, with a small underestimation of energy expenditure by the kmsMovesensor. The averaged total difference is $121 \mathrm{kcal}$ or $7 \%$.

The intraclass correlation analysis revealed a high correlation between the test readings of the kmsMove-sensor and indirect calorimetry for a measuring time of $100 \mathrm{~min}(0.82$ $(0.38-0.96) ; p=0.003)$ and for a time period of an average of $7 \mathrm{~h}(0.81(0.22-0.97) ; p=0.01)$. The Bland-Altman analysis for the time period of $100 \mathrm{~min}$ revealed a bias (difference of the means) of $4.3 \mathrm{kcal}$ and limits of agreement of -94.3 and $102.9 \mathrm{kcal}$. As one can see from the Bland-Altman plot (Fig. 1), all measured differences lie between the limits of agreement.

For the time period of an average of $7 \mathrm{~h}$, the BlandAltman analysis showed a bias of $-14.0 \mathrm{kcal}$ and limits of agreement of -320.0 and $292.0 \mathrm{kcal}$. All measured differences lie between the limits of agreement as well (Fig. 2).

\section{Discussion}

The total averaged difference of $35 \mathrm{kcal}$ or $8 \%$ over a measuring period of $100 \mathrm{~min}$ as well as the total averaged

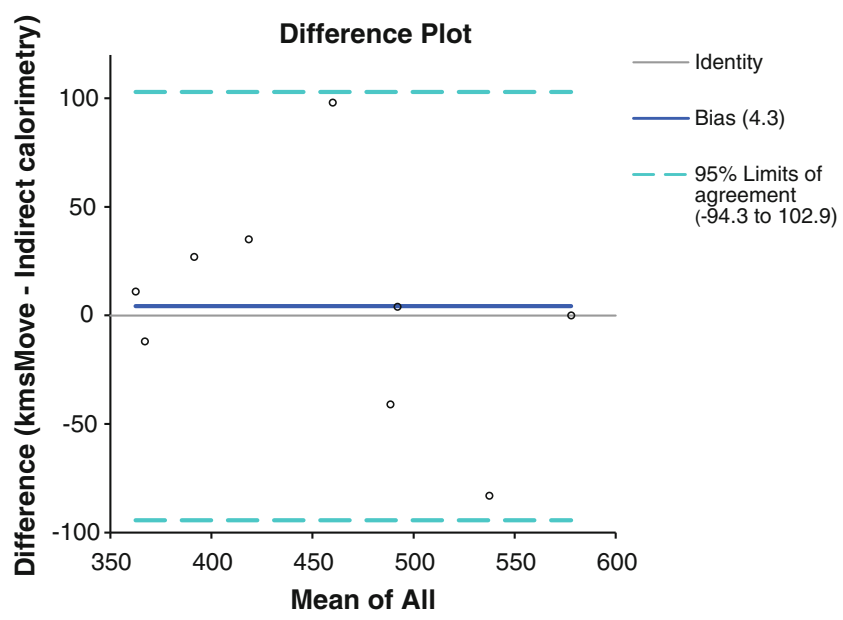

Fig. 1 Bland-Altman plot for the time period of $100 \mathrm{~min}$

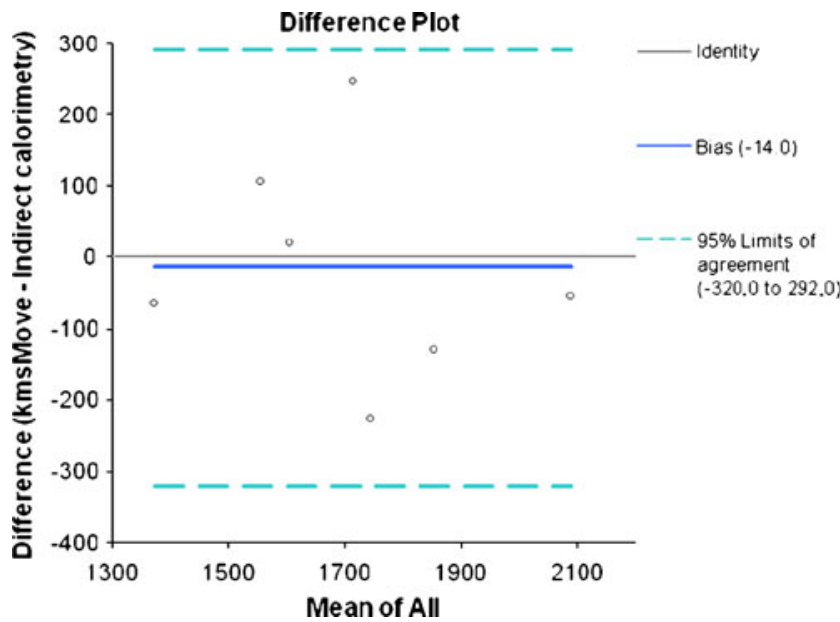

Fig. 2 Bland-Altman plot for the time period of an average of $7 \mathrm{~h}$ 
difference of $121 \mathrm{kcal}$ or $7 \%$ over a measuring time at an average of $7 \mathrm{~h}$ seems to be quite acceptable. Especially if one keeps in mind that the reference measure indirect calorimetry is considered a "gold standard" for measuring energy expenditure but is not appropriate for measurements in everyday life. As the intraclass correlation demonstrates, there is a high correlation between the test readings of the kmsMovesensor and indirect calorimetry over a measuring time of $100 \mathrm{~min}$ as well as over an average of $7 \mathrm{~h}$. The bias of $4.3 \mathrm{kcal}$ over a measuring time of $100 \mathrm{~min}$ and a bias of $-14 \mathrm{kcal}$ at an average of $7 \mathrm{~h}$ can be neglected in reference to the measurement duration and confirms the acceptable agreement between the two devices. Nevertheless, there are large limits of agreement for both measurement durations. Potential causes for the variations of the two devices could be the basic metabolic rate and static activities such as gymnastics or bicycle ergometer training, since these activities contain only slight movements of the hip and energy expenditure of arm and leg movements cannot be adequately assessed. The partially wide ranges of energy expenditure between the subjects are likely due to their different rehabilitation programs, since some of the subjects performed a lot of exercise therapy activities while others did more relaxation activities over their measuring period.

To establish a comparison with other accelerometers, we searched Medline databases for validation studies with the following key words: "accelerometry", "accelerometer", "validity", "validation", "energy expenditure", and "energy consumption". Our criteria for further consideration were indirect calorimetry as reference measure, comparable statistical analyses, adult subjects, no laboratory assessments, and similar measurement durations. In comparison to other accelerometers, the kmsMove-sensor provides relatively good test readings. Bassett et al. (2000) found correlations of $r=0.33-0.62$ for several accelerometers (CSA 7164, Caltrac, Kenz Selects 2, Yamax SW-701) [11]. Welk et al. (2000) validated three different physical activity monitors (CSA, Tritrac, Biotrainer) by letting the subjects complete choreographed routines of different lifestyle activities. The mean correlation coefficient for the three accelerometers compared to indirect calorimetry was 0.55 [12]. A study of Berntsen et al. (2010) compared four accelerometers (SenseWear $\mathrm{Pro}_{2}$, ikcal, ActiGraph, ActiReg) to indirect calorimetry and found ICCs of 0.73 (0.44-0.88), $0.71(0.41-0.87)$, both $p<0.001$ and of 0.55 $(0.16-0.79 ; p=0.005)$, and $0.47(0.02-0.75 ; p=0.004)$, respectively. The mean differences and limits of agreement from Bland-Altman analyses were $-50.0 \mathrm{kcal}(-446.7$ and 346.7), -111.1 kcal (-409.3 and 187.1), -43.4 kcal (-304.5 and 217.6), and $-33.9 \mathrm{kcal}$ (-299.1 and 231.3), respectively for ActiGraph, ActiReg, SenseWear $\mathrm{Pro}_{2}$, and ikcal [13]. Regarding the results of the statistical analyses from the validation studies of these accelerometers, the kmsMove- sensor seems to possess a better measurement accuracy in comparison to the above-mentioned motion sensors. When drawing this conclusion, one has to keep in mind that the sample size of this study was very small and the participants consisted only of men. Furthermore, the rehabilitation setting is somewhat artificial because the subjects are bound to a certain program comprising several activities that are not typical for the daily lifestyle of the subjects (e.g., relaxation therapy, snowshoeing). So these results are not generalizable for other groups of people (e. g., women, children), and they are only transferable in a restricted manner to activities of daily living.

\section{Conclusion}

Taking into account the discussed limitations of the study, the kmsMove-sensor seems to be a device that is quite adequate for assessing energy expenditure. The obtained measurement accuracy of the kmsMove-sensor is likely due to the activity recognition and the resulting selection of one of five algorithms to compute energy expenditure. As we showed in this study, the sensor possesses good applicability in a rehabilitation context. Possible other fields of application might be prevention or health promotion studies as well as epidemiological studies. In this context, further studies should investigate larger and mixed samples within naturalistic settings containing various typical activities of daily living.

\section{References}

1. Paffenbarger RS, Blair SN, Lee IM et al (1993) Measurement of physical activity to assess health effects in free living populations. Med Sci Sports Exerc 25:60-70

2. Sirard JR, Pate RR (2001) Physical activity assessment in children and adolescents. Sports Med 31:439-454

3. Myrtek M, Foerster F, Brügner G (2001) Freiburger monitoring system (FMS). Peter Lang, Frankfurt/Main

4. Crouter SE, Churilla JR, Bassett DR Jr (2006) Estimating energy expenditure using accelerometers. Eur J Appl Physiol 98:601-612

5. Trost SG, Way R, Okely AD (2006) Predictive validity of three ActiGraph energy expenditure equations for children. Med Sci Sports Exerc 38:380-387

6. Jatobá L, Grossmann U, Kunze C, et al (2008) Context-aware mobile health monitoring: evaluation of different pattern recognition methods for classification of physical activity. Proceedings IEEE EMBC 2008: 30th Annual International Conference of the IEEE Engineering in Medicine and Biology Society, Vancouver, British Columbia, Canada, pp 5250-5253

7. Jatobá L, Großmann U, Ottenbacher J, et al (2007) Obtaining energy expenditure and physical activity from acceleration signals for context-aware evaluation of cardiovascular parameters. IFMBE Proceedings CLAIB 2007: IV Latin American Congress on Biomedical Engineering 2007, Bioengineering Solutions for Latin America Health, Margarita Island, Venezuela, vol 18, pp 475-479 
8. de Marées H (2003) Sportphysiologie. SPORTVERLAG Strauß, Köln

9. Acheson KJ (1988) Theory, assumptions and limitations of calculating energy expenditure and substrate utilization from respiratory exchange data. In: Workshop on Methodological Questions on Indirect Calorimetry, 1986, Salzburg, Austria. W. Zuckschwerdt Verlag, Salzburg

10. Bland JM, Altman DG (1986) Statistical methods for assessing agreement between two methods of clinical measurement. Lancet $8: 307-310$
11. Bassett DR Jr, Ainsworth BE, Swartz AM et al (2000) Validity of four motion sensors in measuring moderate intensity physical activity. Med Sci Sports Exerc 32:S471-S480

12. Welk GJ, Blair SN, Wood K et al (2000) A comparative evaluation of three accelerometry-based physical activity monitors. Med Sci Sports Exerc 32:S489-S497

13. Berntsen S, Hageberg R, Aandstad A et al (2010) Validity of physical activity monitors in adults participating in free-living activities. Br J Sports Med 44:657-664 